\title{
GAP ACCEPTANCE FOR LEFT TURNS FROM THE MAJOR ROAD AT UNSIGNALIZED INTERSECTIONS
}

\author{
Hongmei Zhou ${ }^{1}$, John N. Ivan², Per E. Gårder ${ }^{3}$, Nalini Ravishanker ${ }^{4}$ \\ ${ }^{1}$ College of Transportation \& Logistics, Dalian University of Technology, China \\ ${ }^{2}$ Dept of Civil and Environmental Engineering, University of Connecticut, United States \\ ${ }^{3}$ Dept of Civil and Environmental Engineering, University of Maine, United States \\ ${ }^{4}$ Dept of Statistics, University of Connecticut, United States
}

Submitted 18 November 2013; resubmitted 18 January 2014; accepted 26 May 2014; first published online 11 July 2014

\begin{abstract}
This paper attempts to identify factors that may influence the gap acceptance behavior of drivers who turn left from the major road at unsignalized intersections. Drivers' accepted and rejected gaps as well as their age and gender were collected at six unsignalized intersections with both two and four lanes on the major road, with and without the presence of a Left-Turn Lane (LTL), and with both high and low Speed Limits (SLs). Whether or not a driver accepts a given gap was considered as a binary decision and correlated logit models were used to estimate the probability of accepting a gap. Models with different factors were tested and the best model was selected by the quasi-likelihood information criterion. The gap duration, the number of rejected gaps, the mean and total time interval of the rejected gaps and the gender of the driver were all significant in explaining the variation of the gap acceptance probability, whereas the number of lanes of the major road, the presence of LTL, the SL and the driver's age category were not. Gap acceptance probability functions were determined based on the best model, including both the factors of the number of rejected gaps and the mean time interval of the rejected gaps. As the values of these two factors increase, the probability of accepting a given gap rises up. The developed model can be further applied in practice to improve the analysis of traffic operations and capacity at unsignalized intersections.
\end{abstract}

Keywords: unsignalized intersection; left turn; gap acceptance; correlated logit model; traffic operations.

\section{Introduction}

At two-way stop-controlled unsignalized intersections, traffic of lower priority must yield to traffic of higher priority. For example, drivers on the major road turning left must seek for gaps between oncoming vehicles to decide when it is safe and comfortable to execute the turning maneuver. Consequently, capacity analysis of unsignalized intersection is founded upon a clear understanding of the interactions between drivers with different levels of priority, of which gap acceptance theory is an important part.

A driver's critical gap cannot be directly measured, but lies between the largest rejected gap and smallest accepted gap by a driver. Many researchers have proposed different definitions and estimation procedures for the critical gap parameter. According to the Highway Capacity Manual (2000) - HCM, the critical gap is the minimum time interval between vehicles in a traffic stream that is acceptable for the driver to complete a conflicting maneuver. It is estimated by the maximum likelihood method assuming that drivers are homogeneous and consistent, i.e. they are expected to all behave the same way and will accept all gaps that are larger than the critical gap and reject all smaller gaps. The base values of the critical gap and follow-up time (the amount of time needed for a second vehicle to tag along behind the lead vehicle in addition to the critical gap) for passenger cars are provided for all non-priority manoeuvres at an unsignalized intersection. For permitted left turns from the major street, the base value of critical gap is $4.1 \mathrm{~s}$ for both two-lane and four-lane major streets. Adjustments are made to account for the proportion of heavy vehicles and the approach grade. Once the critical gap is determined according to the procedures in the HCM,

Corresponding author: Hongmei Zhou

E-mail: jennifer_zhm@hotmail.com 
the potential capacity of each minor traffic stream can be computed using the gap acceptance model:

$$
c=v \cdot \frac{\exp \left(-\frac{v \cdot t_{c}}{3600}\right)}{1-\exp \left(-\frac{v \cdot t_{f}}{3600}\right)},
$$

where: $c$ - potential capacity of the minor movement [veh/h]; $v$ - conflicting flow rate for the subject movement $[\mathrm{veh} / \mathrm{h}] ; t_{c}$ - critical gap for the subject movement; $t_{f}$ - follow-up time for the subject movement. Smaller values of critical gap and follow-up time result in increasing capacity; larger values result in decreasing capacity.

As a commonly accepted method which has been applied worldwide, the HCM method of estimating the critical gap has some limitations. First, the HCM method, along with many others, treats the critical gap as a deterministic single value for accepting or rejecting a gap, which may not conform to the drivers' behavior in reality. Alternatively, other researchers have proposed probabilistic methods to model drivers' gap acceptance behavior, in which the critical gap is defined as a gap value that fifty percent of drivers would accept, or in other words, the probability of accepting the critical gap is 0.5 (Dissanayake et al. 2002; Yan et al. 2007), and thus the critical gap can be determined by using a gap acceptance probability function. Secondly, while the HCM provides gap values that can be conveniently used in a broad range of conditions, more accurate values of the critical gaps may be obtained by field observation to account for site-specific conditions. When turning left from the major road, such factors as the number of lanes to be crossed, the presence of Left-Turn Lanes (LTLs) on the major road, and the speed of oncoming traffic as well as the traffic density may all affect the critical gap value. For instance, drivers may require longer gaps for crossing two lanes of oncoming traffic than just one lane of traffic; they may feel pressure waiting in the same lane as through vehicles when there are no left-turn lanes and thus accept shorter gaps; they may become more conservative when crossing high speed oncoming traffic; however, they may become impatient waiting for a safe gap from the dense oncoming traffic and take risky behavior. Thus, individual driver's gap acceptance behavior may change with time and conditions. Moreover, the driver's own characteristics, such as age and gender, etc., may also have impact on their gap acceptance behavior.

Consequently, the objective of this study is to investigate through field studies the individual driver's gap acceptance process and develop a model that more complies with the real-world driving behavior. The study focuses on drivers making left turns from the major road at unsignalized intersections and their gap acceptance behavior is expected to be influenced by factors like intersection layout, conflicting traffic flow and driver's own characteristics. The study data were collected at six unsignalized intersections in the State of Connecticut,
USA, with both two and four lanes on the major road, with and without the presence of a LTL, and with both high and low Speed Limits (SLs). Drivers were observed turning left from the major road and their accepted and rejected gaps along with their age and gender were observed. Statistical analysis was conducted to identify factors that are significantly associated with whether or not a gap was accepted. Gap acceptance probability functions were determined, and detailed discussion and conclusions were provided at last.

\section{Literature Review}

Many different methods exist for the estimation of critical gap at unsignalized intersections, which lead to different calculation of capacity. Brilon et al. (1999) provided an overview of the important methods and recommended the maximum likelihood method for practical application. McGowen and Stanley (2012) in a recent study proposed an alternative model for estimating the critical gap, which could yield accurate estimates of the mean critical gap as long as accurate estimates of the major street traffic were given. Vasconcelos et al. (2013) compared different methods to estimate critical headway at roundabouts and suggested that locally calibrated parameters should be used for capacity calculations.

Meanwhile, extensive research using probabilistic and behavioral approaches have been conducted to study the influential factors of drivers' gap acceptance behaviour and found that the critical gap parameter could be dependent on a variety of factors. First, the gap duration was the most obvious factor and the longer the gap, the more likely it would be accepted by drivers (Alexander et al. 2002; Yan et al. 2007; Devarasetty et al. 2012; Zohdy, Rakha 2012; Zohdy et al. 2011, 2010; Beanland et al. 2013). Another factor was the travel speed of the opposing traffic. Davis and Swenson (2004) found that the probability of a driver accepting a gap would increase as the speed of the oncoming vehicle increased, indicating that drivers might underestimate the time-toarrival of vehicles under high-speed environment. Some other researchers investigated the effects of opposing vehicle travel speed and the driver characteristics together. For example, Staplin and Lyles (1991) conducted a study on the effect of vehicle speed on the minimum gap accepted by drivers turning left and found that older drivers were relatively insensitive to vehicle approach speed in left-turn situations. Yan et al. (2007) used a driving simulator to study the effects of major traffic speed and driver age and gender on gap acceptance behavior and demonstrated that older drivers, especially older female drivers are more conservative when executing left turns. This was consistent with the findings by Alexander et al. (2002) that the shorter the gap, the lower the probability of it being accepted by elderly or female drivers compared to non-elderly or male drivers. Whereas age and gender were the most frequently investigated driver characteristics, Pollatschek et al. (2002) defined individual critical gap for risk-loving and cautious drivers and presented a decision model based on evaluation of 
the risk associated with not accepting small gaps against the potential benefit of accepting them. To account for driver differences in gap acceptance behavior, many microscopic simulation programs, such as the widely used software VISSIM, allow the specification of different driver classes.

Previous studies also reported that drivers' gap acceptance behavior related to the traffic conditions, especially to the waiting time at the stop-line. To some extent, the waiting time indirectly reflected the density of the conflicting traffic flow and the longer a driver waited, the more likely he accepted shorter gaps. Polus et al. (2003, 2005) proposed a behavioral approach and showed that the wait time had a significant effect on the critical gap of entry into a roundabout, particularly on gaps in the range of 2 to 5 seconds. Thus roundabout capacity for this range of critical gaps is higher than that proposed by the HCM. Zohdy et al. (2010) pointed out that the acceptable gap duration for permitted left-turn maneuvers decreased as a function of the driver's wait time. Rakha et al. (2011) found that drivers become more aggressive as they waited longer. Devarasetty et al. (2012) showed in their research that total wait time was significant in predicting the probability of accepting or rejecting a gap for a left-turn vehicle from the major road. On the contrary, Kaysi and Abbany (2007) formulated a behavior model that predicted the probability that a driver performed an aggressive manoeuvre as a function of a set of driver and traffic attributes. However, it was found that the total wait time for the driver while waiting for an acceptable gap was of little significance in incurring the forcing behavior. Generally, researchers recommended adopting different critical gap values in different traffic conditions (Cassidy et al. 1995; Devarasetty et al. 2012).

In addition, the impact of inclement weather on gap acceptance behavior has been studied in recent years. It was indicated that the critical gap changed as a function of the rain intensity, and drivers were more conservative during snow than rain (Zohdy et al. 2010, 2011; Zohdy, Rakha 2012; Rakha et al. 2011). Moreover, visibility conditions (day/night) also influence driver's gap acceptance (Dissanayake et al. 2002; Alexander et al. 2002).

When using the probabilistic approach to model drivers' gap acceptance behavior, logistic models were often developed to relate the influence factor to the drivers' gap acceptance decision, and model coefficients could be estimated by a Generalized Linear Model (GLM). The inferences about the regression coefficients were based on the assumption that it approximately followed a normal distribution given the sample size was large. If such assumption did not hold, alternative method might be used to solve the model parameters, such as Bayesian or bootstrap approaches (Rakha et al. 2011). The major advantage of these methods was that they might be applied with relatively small sample size, not requiring the assumption of the distribution of the parameters.

Regarding the research data, in the literature researchers generally used either experiments by driving simulators (Alexander et al. 2002; Yan et al. 2007; Bean- land et al. 2013) or field observations (Rakha et al. 2011; Davis, Swenson 2004; Polus et al. 2003, 2005) to obtain drivers' gap acceptance data. The merit of using driving simulator is obvious that the driving experiments can be elaborately designed and controlled, and the results are repeatable. However, data collected from the field may better reflect the real-world behavior, and thus yield more valid results.

Furthermore, from the safety perspective gap acceptance is a critical maneuver for drivers turning or crossing the intersection and poses potential crash risks. Thus, Intersection Decision Supporting (IDS), either a road-side system or an in-vehicle system, has been investigated by researchers to assist drivers in making safer gap acceptance decisions (Laberge et al. 2006; Creaser et al. 2007; Becic et al. 2012; Zohdy, Rakha 2012). Varying levels of dynamic information about the traffic conditions may be presented to drivers through IDS and exerts different effects on drivers' gap selection. The provision of advisory information by IDS should be based on a comprehensive study of the potential influence factors of drivers' gap acceptance behavior.

In this study, the method of field observations was used aiming to further contribute to the understanding of the factors affecting the gap acceptance behavior of drivers making left turns from the major road at unsignalized intersections. Specifically, the effects of the presence of a LTL, the number of lanes being crossed and the speed of the conflicting traffic are being investigated as well as the driver's age and gender. The conflicting traffic condition is an important factor because the gap distribution depends on it. However, it is difficult to determine an appropriate microscopic variable to directly represent the varying traffic condition during an individual driver's gap selection procedure. In this study, the number of rejected gaps, the mean value of the rejected gaps and the total interval of the rejected gaps by each driver are examined. These variables are expected to reflect the traffic condition better than the wait time that has been used in most previous studies. Thus, they may be more closely related to the driver's gap acceptance behavior.

\section{Study Design}

The first step of this study was to select the intersections for field observation. For consistency of driver behavior, the focus of the study was at unsignalized intersections with stop control only on the minor road approaches. Both high-speed locations (SLs $\geq 45 \mathrm{mph}$ ) and low speed locations (SLs $<45 \mathrm{mph}$ ) were included to account for differences of gap-acceptance behavior under different speed levels. Locations with two and four traffic lanes on the main road, and with and without a LTL for the subject maneuver, were also included. This resulted in a total of eight different possible combinations of these features. To select appropriate sites, it was critical that the volume of the through traffic and left-turning traffic at each location be high enough so that there was a need for drivers to wait and evaluate whether the gap 
was suitable for the turning maneuver. In addition, the locations selected should be convenient for the setup of cameras that were used to assist in data collection, and locations with noticeable grades should also be avoided.

The Connecticut Department of Transportation (ConnDOT) photolog system, a collection of digital video images of the entire State-maintained road network, was used to locate candidate intersections. Site visits were also conducted to evaluate the traffic-related criteria. However, no intersections with both high SL and LTLs were found because such intersections either were signalized or had very low through and left turning traffic volumes. Finally, six intersections were chosen, with one for each of the six intersection types, coded as $2 \mathrm{NH}, 2 \mathrm{NL}, 4 \mathrm{NH}, 4 \mathrm{NL}, 2 \mathrm{YL}$, and $4 \mathrm{YL}$. The codes were defined as follows:

- 2 - 2 lane major road;

- 4 - 4 lane major road;

- N - without LTL;

- Y - with LTL;

- H - high SL ( $\geq 45 \mathrm{mph})$;

- L - low SL $(<45 \mathrm{mph})$.

Digital video recordings were made of drivers turning left from the major road and their gap acceptance behavior at each intersection. The video was processed later in the lab to extract data needed such as the time when the left turning vehicle arrived, the gap length in the oncoming traffic, and whether or not the gap was accepted by the driver. Drivers' age and gender were observed on site (without attracting the drivers' attention) since the video was intentionally not detailed enough to identify drivers. The arrival time and vehicle color and type were also noted for each driver in order to easily identify the vehicle later in the video. The drivers' information was then linked with their gap acceptance observations. Lags were not observed in this study.
In order to get reasonably accurate age estimates, two observers were trained in judging a person's age prior to the observation. About 40 volunteers who were willing to reveal their ages were used for this training. The observers were asked to estimate the person's age and the real age was revealed immediately afterwards. It appears that the estimates made later in the training period were more accurate than earlier ones, indicating that observers were improving on their ability to estimate age differences during this process. In more than $80 \%$ of these age observation tests, the estimated age by the observers was within a 5-year range of the person's true age.

In this paper, to investigate the possible age differences in gap acceptance behavior, drivers were classified into four age categories:
1) age $<25$;
2) $25 \leq$ age $<45$;
3) $45 \leq$ age $<65$;
4) age $\geq 65$.

Drivers of age older than 65 are commonly accounted as elderly drivers in many countries, who are expected to exhibit more conservative behavior compared to other drivers. For drivers younger than 65 , we further classified them into three groups, representing young, young to middle-aged and middle-aged drivers, respectively, and behavior differences among them will also be examined in this study. The data recorded for each gap include the gap length (in seconds), the age and gender of the driver, the attributes of the intersection where this gap was observed, and whether or not the gap was accepted. Then, the number of rejected gaps, the mean time interval of the rejected gaps and the total time interval of the rejected gaps were also calculated. Table 1 lists the number of left turning drivers and the number of gaps observed at each intersection, along

Table 1. Number of drivers and gaps observed by intersection

\begin{tabular}{|c|c|c|c|c|c|c|c|}
\hline Intersection & Lane & LTL & SL $[\mathrm{mph}]$ & Drivers & Gaps & $V 1$ & $V 2$ \\
\hline 1 & 2 & $\mathrm{~N}$ & $45(\mathrm{H})$ & 54 & 103 & 204 & 220 \\
\hline 2 & 2 & $\mathrm{~N}$ & $35(\mathrm{~L})$ & 57 & 144 & 470 & 407 \\
\hline 3 & 4 & $\mathrm{~N}$ & $45(\mathrm{H})$ & 250 & 1771 & 874 & 832 \\
\hline 4 & 4 & $\mathrm{~N}$ & $30(\mathrm{~L})$ & 284 & 1156 & 647 & 717 \\
\hline 5 & 2 & $\mathrm{Y}$ & $35(\mathrm{~L})$ & 261 & 982 & 562 & 600 \\
\hline 6 & 4 & $\mathrm{Y}$ & $35(\mathrm{~L})$ & 61 & 496 & 1365 & 1399 \\
\hline
\end{tabular}

Table 2. Quartiles of accepted and rejected gap distributions by intersection [s]

\begin{tabular}{|c|c|c|c|c|c|c|}
\hline \multirow{2}{*}{ Intersection } & \multicolumn{3}{|c|}{ Accepted gaps } & \multicolumn{3}{|c|}{ Rejected gaps } \\
\hline & $25 \%$ & $50 \%$ & $75 \%$ & $25 \%$ & $50 \%$ & $75 \%$ \\
\hline $2 \mathrm{NH}$ & 7.5 & 10 & 10 & 1.4 & 2.0 & 3.1 \\
\hline $2 \mathrm{NL}$ & 7.0 & 10 & 10 & 1.0 & 1.6 & 3.3 \\
\hline $4 \mathrm{NH}$ & 5.4 & 7.5 & 10 & 0.8 & 1.2 & 2.0 \\
\hline $4 \mathrm{NL}$ & 6.4 & 10 & 10 & 1.0 & 1.7 & 2.7 \\
\hline $2 \mathrm{YL}$ & 6.2 & 8.7 & 10 & 1.1 & 1.8 & 2.8 \\
\hline $4 \mathrm{YL}$ & 4.8 & 5.7 & 8.0 & 0.8 & 1.6 & 2.4 \\
\hline
\end{tabular}


with the intersection characteristics. One-hour traffic counts of the subject road segment $V 1$ and the oncoming road segment $V 2$ were also obtained at each intersection for reference.

Since a driver may possibly reject several gaps before accepting one to execute the turning maneuver, the total number of gaps observed is far more than the number of drivers. A total of 967 drivers with 4652 gaps at six intersections were observed, about five gaps per driver on average. A wide variety of gap lengths was observed, with many much longer than $10 \mathrm{~s}$ (the highest value we tabulated), and also many gaps shorter than $2 \mathrm{~s}$, and all values in between. Table 2 lists statistics of the gaps observed for each intersection.

\section{Methodology}

\subsection{Correlated Logit Model}

Whether or not a driver accepts or rejects a given gap could be considered as a binary decision, and logit models are often used in this case to identify variables associated with binary decisions. Observing the data, the total number of gaps is more than the number of drivers, since a driver may reject several gaps before he finally accepts one. In other words, driver $i$ may come across $n_{i}$ gaps, where $n_{i} \geq 1$. If the response variable is coded as $y_{i j}=1$ when the $j$ th gap $\left(j=1, \ldots, n_{i}\right)$ for driver $i$ is accepted and $y_{i j}=0$ when it's rejected, it's obvious that $y_{i j}\left(j=1, \ldots, n_{i}\right)$ are not independent but correlated binary random variables. Ordinary binary models cannot explain the correlation among these dependent variables. Instead, a correlated binary model was used to identify factors that have significant effects on gap acceptance decisions. The model was in the form of:

$$
p_{i j}=\frac{1}{1+\exp \left(-x_{i j}^{\prime} \cdot \beta\right)},
$$

where: $p_{i j}$ is the probability that driver $i$ accept gap $j$, $j=1, \ldots, n_{i} ; x_{i j}^{\prime}$ is the vector of the covariates of the $j$ th gap for driver $i, j=1, \ldots, n_{i} ; \beta$ is the vector of regression coefficients.

The factors under study included the gap length, the number of rejected gaps $n_{r e j}$, the mean $m_{r e j}$ and total time interval $t_{r e j}$ of the rejected gaps, the number of lanes on the major road, the presence or absence of a LTL, the SL, as well as the driver's age category and gender. Except the first four variables, others were all categorical variables, as coded in Table 3 . The age category had four levels so 3 indicator variables were created, using the age category 4 as the reference level. The variable age 1 was coded as 1 if the driver was in age category 1 and 0 otherwise, and age 2 and age 3 was coded in the same manner. Sample records were shown in Table 4. It showed a female driver rejecting 4 gaps $(1.9,3.8,4$, and $6.1 \mathrm{~s})$ and accepting the fifth gap (7 s). Thus, there were five records for this driver, and in each record, the number of rejected gaps, the mean and total time interval of the rejected gaps were calculated. Models were estimated using GLM with binary response in SAS ${ }^{\bullet}$. Generalized
Table 3. Categorical variable values and coding in the model

\begin{tabular}{|c|c|c|c|}
\hline Variable & Value & Frequency & Coding \\
\hline \multirow{3}{*}{ Lane } & 2 & 1229 & 1 \\
\cline { 2 - 4 } & 4 & 3423 & 0 \\
\hline \multirow{3}{*}{ LTL } & $\mathrm{N}$ & 3174 & 1 \\
\cline { 2 - 4 } & $\mathrm{Y}$ & 1478 & 0 \\
\hline \multirow{2}{*}{ SL } & $\mathrm{H}$ & 1874 & 1 \\
\cline { 2 - 4 } & $\mathrm{L}$ & 2778 & 0 \\
\hline \multirow{3}{*}{ Age1 } & age $<25$ & 242 & 1 \\
\cline { 2 - 4 } & otherwise & & 0 \\
\hline \multirow{3}{*}{ Age2 } & $25 \leq$ age $<45$ & 2097 & 1 \\
\cline { 2 - 4 } & otherwise & & 0 \\
\hline \multirow{2}{*}{ Age3 } & $45 \leq$ age $<65$ & 1434 & 1 \\
\cline { 2 - 4 } & otherwise & & 0 \\
\hline \multirow{2}{*}{ Gender } & female $(\mathrm{F})$ & 2463 & 1 \\
\cline { 2 - 4 } & male $(\mathrm{M})$ & 2189 & 0 \\
\hline
\end{tabular}

Table 4. Sample data records for one driver

\begin{tabular}{|c|c|c|c|c|c|c|c|c|c|c|}
\hline ID & Lane & LTL & SL & Age & Gender & Gap & $n_{r e j}$ & $t_{r e j}$ & $m_{r e j}$ & $y$ \\
\hline 35 & 2 & $\mathrm{~N}$ & $\mathrm{~L}$ & 2 & $\mathrm{~F}$ & 1.9 & 0 & 0 & 0.00 & 0 \\
\hline 35 & 2 & $\mathrm{~N}$ & $\mathrm{~L}$ & 2 & $\mathrm{~F}$ & 3.8 & 1 & 1.9 & 1.90 & 0 \\
\hline 35 & 2 & $\mathrm{~N}$ & $\mathrm{~L}$ & 2 & $\mathrm{~F}$ & 4 & 2 & 5.7 & 2.85 & 0 \\
\hline 35 & 2 & $\mathrm{~N}$ & $\mathrm{~L}$ & 2 & $\mathrm{~F}$ & 6.1 & 3 & 9.7 & 3.23 & 0 \\
\hline 35 & 2 & $\mathrm{~N}$ & $\mathrm{~L}$ & 2 & $\mathrm{~F}$ & 7 & 4 & 15.8 & 3.95 & 1 \\
\hline
\end{tabular}

Estimating Equations (GEE) were used to account for the correlation among the response variable $y_{i j}$ within the same driver $i$. In SAS, the GEE solution is requested by using the REPEATED statement in the GENMOD procedure.

\subsection{Generalized Estimating Equations}

GEE is an extension of GLM in that they allow adjusting for correlation among the responses. Data are modeled using the same link function, but the covariance structure of the correlated measurement must also be modeled. Let $y_{i j}, j=1, \ldots, n_{i}, i=1, \ldots, K$, represent the $j$ th measure on the $i$ th subject, with corresponding mean $\mu_{i j}$, and let $R_{i}(\alpha)$ be a $n_{i} \times n_{i}$ working correlation matrix that is specified by the vector of parameters $\alpha$. The covariance matrix of $Y_{i}$ can then be modeled as:

$$
V_{i}=\phi \cdot A_{i}^{\frac{1}{2}} \cdot R(\alpha) \cdot A_{i}^{\frac{1}{2}},
$$

where: $A_{i}$ is a $n_{i} \times n_{i}$ diagonal matrix with $\operatorname{Var}\left(\mu_{i j}\right)$ as the $j$ th diagonal element and $\phi$ is the dispersion parameter. Many correlation structures are available, including fixed, AutoregRessive(1), exchangeable, independent, $m$-dependent, and unstructured. When the structure is autoregressive $\mathrm{AR}(1)$, the correlation between $y_{i j}$ and $y_{i, j+t}$ is:

$$
\operatorname{Corr}\left(y_{i j}, y_{i, j+t}\right)=\alpha^{t} \text { for } t=0,1,2, \ldots, n_{i}-j,
$$

where: there are missing data, i.e., $y_{i k}$ is missing for all $j \geq k$, the GENMOD procedure can estimate the work- 
ing correlation from data by using the all available pairs method, in which all nonmissing pairs of data are used in the moment estimators of the working correlation parameters. The resulting covariances and standard errors are valid under the Missing Completely At Random (MCAR) assumption.

The fitting algorithm for GEE is not a likelihoodbased method of estimation, so inference measures based on likelihood are not applicable to GEE methods. First, it computes an initial estimate of vector of regression parameters $\beta$ with an ordinary generalized linear model and then the working correlations matrix $R_{i}(\alpha)$ is computed based on the standardized residuals; covariance is estimated by Eq. (3); $\beta$ is then updated by:

$$
\begin{aligned}
& \beta_{r+1}=\beta_{r}+\left(\sum_{i=1}^{K} \frac{\partial \mu_{i}^{\prime}}{\partial \beta} \cdot V_{i}^{-1} \cdot \frac{\partial \mu_{i}}{\partial \beta}\right)^{-1} \times \\
& \left(\sum_{i=1}^{K} \frac{\partial \mu_{i}^{\prime}}{\partial \beta} \cdot V_{i}^{-1} \cdot\left(Y_{i}-\mu_{i}\right)\right),
\end{aligned}
$$

and then iterated until convergence.

Statistics that are helpful in assessing the goodness of fit of an ordinary generalized linear model (i.e. how well the model fits the observations) is not applicable when the GEE method has been used. The Quasi-likelihood Information Criterion (QIC) as a modification of the Akaike Information Criterion (AIC), and $\mathrm{QIC}_{\mathrm{u}}$ as an approximation to QIC are applied to models fitted with GEE. It is noted that QIC is appropriate for selecting regression models and working correlations, whereas $\mathrm{QIC}_{\mathrm{u}}$ is appropriate only for selecting regression models.

\section{Results and Discussion}

\subsection{Modeling Results}

As shown in Tables 5-7, three categories of models were estimated, in which the effects of the total time interval of the rejected gaps, the number of rejected gaps, and the mean time interval of the rejected gaps were investigated, respectively. For each category, first a model with all of the main factors and two-way interactions as well as possible three-way interactions was fitted to the data. All of the non-significant interaction terms were removed and a new model with the main factors and the remaining interactions was then estimated. Then, the non-significant interaction terms and main factors identified in the model were removed and the model with the remaining variables was fitted. This procedure continued until all the variables in the model were significant (none of the interactions were significant).

For the purpose of analyzing the effects of the factors, three models were displayed in each category. For example, in Table 5, the intercept, gap duration, the total time interval of rejected gaps, the gender were all significant through Models 1-3, but the number of lanes, the presence of LTL, the SL and the driver's age category were not. Comparing the three models, the parameter estimates for the same variable did not show much dif- ference, indicating the consistency of the results. Similar results were obtained in Models 6-7, in which the number of lanes, the presence of LTL, the SL and the driver's age category were generally not significant. The models including the number of rejected gaps (Table 6) showed lower QIC and $\mathrm{QIC}_{\mathrm{u}}$ compared with models with the total time interval of the rejected gaps (Table 5) and the mean time interval of the rejected gaps (Table 7). This may indicate that the number of rejected gaps can better explain the variation of the driver's gap acceptance behavior than the others. The parameter estimates were positive, meaning that with an increase in the number of rejected gaps, the probability of accepting a given gap went up accordingly.

In Table 8 , three simplified models were presented with those insignificant factors removed. In Model 11, the number of rejected gaps and the mean time interval of the rejected gaps were fitted simultaneously. Compared with Model 12, including only the number of rejected gaps, Model 11 had a little bit lower values of QIC and $\mathrm{QIC}_{\mathrm{u}}$. The parameter estimates for the gap, the number of rejected gaps, and the mean time interval of the rejected gaps were all positive, suggesting that as the values of these variables increased, the probability of drivers accepting the gap increased; whereas that for the female was negative, meaning that the probability of accepting a gap is lower for female drivers. With the best fit statistics, Model 12 was finally selected as the gap acceptance model for further investigation.

\subsection{Gap acceptance probability function}

By assuming an equal percent of male and female drivers turning left $($ Gender $=0.5)$, the gap acceptance probability function can be obtained as shown in Eq. (6):

$$
p=\frac{1}{1+\exp \left(8.6546-1.671 \cdot G a p-0.1496 \cdot m_{r e j}-0.0709 \cdot n_{r e j}\right)},
$$

where: $p$ is the probability for a driver to accept a given gap; Gap is the given gap duration [s]; $m_{r e j}$ is the mean duration of the rejected gaps; $n_{r e j}$ is the number of rejected gaps.

Fig. 1 displays the gap acceptance probability curve for different number of rejected gaps $n_{r e j}$. It shows that the probability of accepting a given gap is increasing as the number of rejected gaps rises. For instance, in Fig. 1a, when the mean time interval of the rejected gaps is $1 \mathrm{~s}$, the probability of accepting a 4-second gap is 0.14 for $n_{r e j}=1$, while it is 0.32 for $n_{r e j}=15$. To examine the effects of the mean time interval of the rejected gaps, Fig. 2 shows that as values of this factor increase, the probability goes up accordingly. For instance, in Fig. 2a when only one gap is rejected, the probability of accepting a 4-second gap is 0.14 for $m_{r e j}=1$, and this probability increases to 0.21 for $m_{r e j}=4$. Similarly, in Fig. $2 \mathrm{~b}$ with fifteen gaps rejected, this probability increases from 0.31 to 0.42 . 
Table 5. Parameter estimates of the models on total time interval of rejected gaps

\begin{tabular}{|c|c|c|c|c|c|c|}
\hline \multirow{2}{*}{ Parameter } & \multicolumn{2}{|c|}{ Model 1} & \multicolumn{2}{|c|}{ Model 2} & \multicolumn{2}{|c|}{ Model 3} \\
\hline & Estimate & S.E. & Estimate & S.E. & Estimate & S.E. \\
\hline Intercept & $-7.9192^{\star \star}$ & 0.3898 & $-7.9497^{\star *}$ & 0.4001 & $-8.0023^{\star *}$ & 0.4037 \\
\hline Gap & $1.6730^{\star \star}$ & 0.0801 & $1.6546^{\star \star}$ & 0.0795 & $1.6545^{\star \star}$ & 0.0797 \\
\hline Two-lane & -0.3855 & 0.2078 & & & & \\
\hline No LTL & & & -0.0344 & 0.1922 & & \\
\hline High SL & & & & & 0.0639 & 0.2135 \\
\hline Total time of rejected gaps & $0.0285^{\star}$ & 0.0128 & $0.0303^{*}$ & 0.0129 & $0.0304^{*}$ & 0.0127 \\
\hline Age $<25$ & -0.3055 & 0.5727 & -0.2681 & 0.5615 & -0.2808 & 0.5659 \\
\hline $25 \leq$ age $<45$ & -0.1285 & 0.2863 & -0.1254 & 0.2833 & -0.1235 & 0.2858 \\
\hline $45 \leq$ age $<65$ & 0.1801 & 0.2907 & 0.1858 & 0.2893 & 0.1857 & 0.2895 \\
\hline Age $\geq 65$ & 0 & & 0 & & 0 & \\
\hline Female & $-0.6131^{\star *}$ & 0.1877 & $-0.6319^{\star *}$ & 0.1871 & $-0.6126^{\star *}$ & 0.1943 \\
\hline Male & 0 & & 0 & & 0 & \\
\hline QIC & \multicolumn{2}{|c|}{1016.4946} & \multicolumn{2}{|c|}{1020.7365} & \multicolumn{2}{|c|}{1021.0679} \\
\hline $\mathrm{QIC}_{\mathrm{u}}$ & \multicolumn{2}{|c|}{1011.0271} & \multicolumn{2}{|c|}{1015.4605} & \multicolumn{2}{|c|}{1015.3988} \\
\hline
\end{tabular}

Notes: ${ }^{*}$ significant at $95 \%$ confidence level; ${ }^{* *}$ significant at $99 \%$ confidence level.

Table 6. Parameter estimates of the models on the number of rejected gaps

\begin{tabular}{|c|c|c|c|c|c|c|}
\hline \multirow{2}{*}{ Parameter } & \multicolumn{2}{|c|}{ Model 4} & \multicolumn{2}{|c|}{ Model 5} & \multicolumn{2}{|c|}{ Model 6} \\
\hline & Estimate & S.E. & Estimate & S.E. & Estimate & S.E. \\
\hline Intercept & $-8.1592^{\star *}$ & 0.3955 & $-8.1734^{\star *}$ & 0.4012 & $-8.2083^{\star *}$ & 0.4050 \\
\hline Gap & $1.6908^{\star *}$ & 0.0813 & $1.6778^{\star *}$ & 0.0809 & $1.6773^{\star \star}$ & 0.0808 \\
\hline Two-lane & -0.3047 & 0.2096 & & & & \\
\hline No LTL & & & -0.0654 & 0.1929 & & \\
\hline High SL & & & & & -0.0269 & 0.2160 \\
\hline Number of rejected gaps & $0.0735^{\star *}$ & 0.0168 & $0.0780^{\star *}$ & 0.0168 & $0.0785^{\star *}$ & 0.0166 \\
\hline Age $<25$ & -0.2866 & 0.5749 & -0.2481 & 0.5654 & -0.2551 & 0.5643 \\
\hline $25 \leq$ age $<45$ & -0.0946 & 0.2925 & -0.0924 & 0.2895 & -0.0876 & 0.2921 \\
\hline $45 \leq$ age $<65$ & 0.2262 & 0.2980 & 0.2348 & 0.2971 & 0.2349 & 0.2973 \\
\hline Age $\geq 65$ & 0 & & 0 & & 0 & \\
\hline Female & $-0.5933^{\star *}$ & 0.1884 & $-0.6120^{\star *}$ & 0.1880 & $-0.6104^{\star *}$ & 0.1955 \\
\hline Male & 0 & & 0 & & 0 & \\
\hline QIC & \multicolumn{2}{|c|}{1004.9710} & \multicolumn{2}{|c|}{1007.3897} & \multicolumn{2}{|c|}{1007.7653} \\
\hline $\mathrm{QIC}_{\mathrm{u}}$ & \multicolumn{2}{|c|}{1000.3514} & \multicolumn{2}{|c|}{1002.9221} & \multicolumn{2}{|c|}{1003.0157} \\
\hline
\end{tabular}

Notes: ${ }^{\star}$ significant at $95 \%$ confidence level; ${ }^{* *}$ significant at $99 \%$ confidence level.

Table 7. Parameter estimates of the models on the mean time interval of rejected gaps

\begin{tabular}{|c|c|c|c|c|c|c|}
\hline \multirow{2}{*}{ Parameter } & \multicolumn{2}{|c|}{ Model 7} & \multicolumn{2}{|c|}{ Model 8} & \multicolumn{2}{|c|}{ Model 9} \\
\hline & Estimate & S.E. & Estimate & S.E. & Estimate & S.E. \\
\hline Intercept & $-7.9495^{\star \star}$ & 0.3799 & $-7.9502^{\star *}$ & 0.3846 & $-8.0438^{\star \star}$ & 0.3945 \\
\hline Gap & $1.6694^{\star *}$ & 0.0789 & $1.6486^{\star *}$ & 0.0776 & $1.6483^{\star *}$ & 0.0777 \\
\hline Two-lane & $-0.4312^{\star}$ & 0.2044 & & & & \\
\hline No LTL & & & -0.0605 & 0.1868 & & \\
\hline High SL & & & & & 0.1087 & 0.2111 \\
\hline Mean time of rejected gaps & $0.1951^{* *}$ & 0.0719 & $0.1906^{* \star}$ & 0.0705 & $0.1947^{\star *}$ & 0.0709 \\
\hline Age $<25$ & -0.3909 & 0.5773 & -0.3426 & 0.5597 & -0.3658 & 0.5693 \\
\hline $25 \leq$ age $<45$ & -0.1575 & 0.2799 & -0.1554 & 0.2772 & -0.1530 & 0.2793 \\
\hline $45 \leq$ age $<65$ & 0.1153 & 0.2896 & 0.1215 & 0.2873 & 0.1208 & 0.2876 \\
\hline Age $\geq 65$ & 0 & & 0 & & 0 & \\
\hline Female & $-0.6276^{\star *}$ & 0.1850 & $-0.6469^{* *}$ & 0.1839 & $-0.6162^{\star \star}$ & 0.1901 \\
\hline Male & 0 & & 0 & & 0 & \\
\hline QIC & \multicolumn{2}{|c|}{1015.8042} & \multicolumn{2}{|c|}{1020.9322} & \multicolumn{2}{|c|}{1021.2827} \\
\hline $\mathrm{QIC}_{\mathrm{u}}$ & \multicolumn{2}{|c|}{1011.4354} & \multicolumn{2}{|c|}{1016.9847} & \multicolumn{2}{|c|}{1016.8030} \\
\hline
\end{tabular}

Notes: ${ }^{\star}$ significant at $95 \%$ confidence level; ${ }^{\star *}$ significant at $99 \%$ confidence level. 
Table 8. Parameter estimates of the models on the mean time interval of rejected gaps

\begin{tabular}{|c|c|c|c|c|c|c|}
\hline \multirow{2}{*}{ Parameter } & \multicolumn{2}{|c|}{ Model 10} & \multicolumn{2}{|c|}{ Model 11} & \multicolumn{2}{|c|}{ Model 12} \\
\hline & Estimate & S.E. & Estimate & S.E. & Estimate & S.E. \\
\hline Intercept & $-8.3009^{\star *}$ & 0.3890 & $-8.3514^{\star *}$ & 0.3862 & $-8.1842^{\star *}$ & 0.3545 \\
\hline Gap & $1.6845^{\star *}$ & 0.0811 & $1.6710^{* *}$ & 0.0806 & $1.6745^{\star *}$ & 0.0800 \\
\hline Two-lane & -0.3102 & 0.2122 & & & & \\
\hline Mean time of rejected gaps & $0.1547^{\star}$ & 0.0753 & $0.1496^{\star *}$ & 0.0749 & & \\
\hline Number of rejected gaps & $0.0659^{\star \star}$ & 0.0170 & $0.0709^{\star *}$ & 0.0169 & $0.0758^{\star \star}$ & 0.0169 \\
\hline Female & $-0.5988^{\star \star}$ & 0.1940 & $-0.6063^{\star *}$ & 0.1935 & $-0.5814^{\star \star}$ & 0.1886 \\
\hline Male & 0 & & 0 & & 0 & \\
\hline QIC & \multicolumn{2}{|c|}{999.3379} & \multicolumn{2}{|c|}{999.5473} & \multicolumn{2}{|c|}{1000.0301} \\
\hline $\mathrm{QIC}_{\mathrm{u}}$ & \multicolumn{2}{|c|}{996.8739} & \multicolumn{2}{|c|}{997.6854} & \multicolumn{2}{|c|}{998.4440} \\
\hline
\end{tabular}

Notes: ${ }^{\star}$ significant at $95 \%$ confidence level; ${ }^{* *}$ significant at $99 \%$ confidence level.

a)

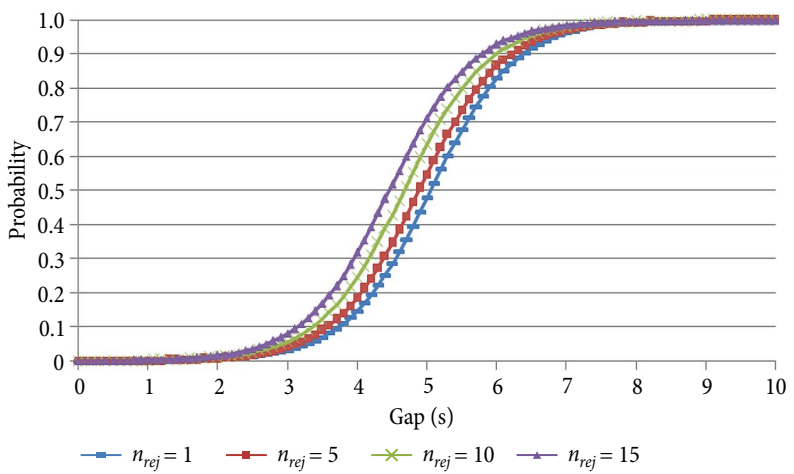

b)

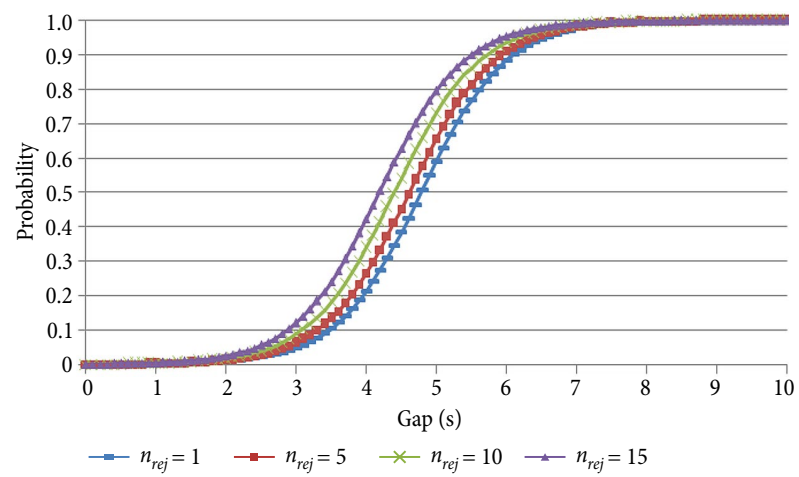

Fig. 1. Gap acceptance probability functions by the number of rejected gaps: $\mathrm{a}-m_{r e j}=1 \mathrm{~s} ; \mathrm{b}-m_{r e j}=4 \mathrm{~s}$

\section{Discussion}

Driver's gap acceptance behavior is of great importance in determining the capacity of minor traffic movement at the intersection and it may be affected by various factors. Firstly, it was expected that drivers would reasonably accept longer gap on four-lane roads than on two-lane major road because it would take more time to cross two lanes of traffic. Also, when there is no LTL, it was expected that drivers would be more willing to accept lower gaps to avoid waiting in a through traffic lane exposed to potential rear end collisions. However, a)

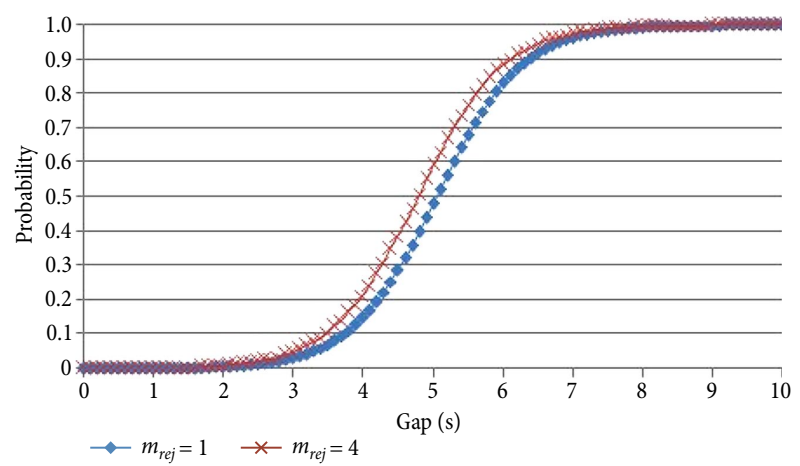

b)

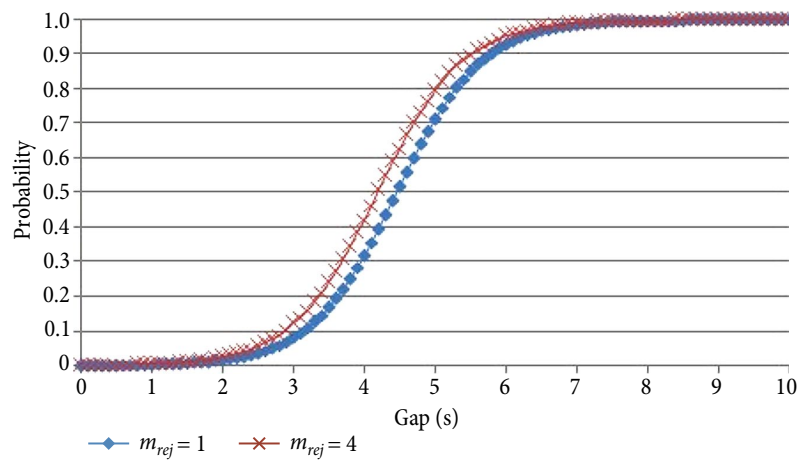

Fig. 2. Gap acceptance probability functions by the mean time interval of rejected gaps: $\mathrm{a}-n_{r e j}=1 ; \mathrm{b}-n_{r e j}=15 \mathrm{~s}$

by observing the collected data, the opposite of these expectations occurred frequently. Initial analysis found that the two-lane road and absence of a left-turn lane were generally correlated with lower intensity of the traffic flow, leading to more conservative behavior in the gap acceptance. On the contrary, four-lane roads and the presence of a left-turn lane are correlated with more intense traffic conditions so that drivers felt it necessary to be more aggressive in order to complete the maneuver. Thus, the different gap acceptance behavior at intersections may be more related to the traffic conditions rather than to the intersection layout. This is reflected in the 
models, where the number of lanes and the presence of the left-turn lane are both insignificant. This result is consistent with HCM, in which the same critical gap value is recommended for both two-lane and four-lane major streets and no differences are recorded between conditions with and without left-turn lanes (Highway Capacity Manual 2000).

As found in the existing research, the gap duration is one of the influential factors for drivers' gap acceptance decision. Basically, longer gaps have higher probability to be accepted if other factors in the proposed model remain the same. Previous findings also showed that drivers were insensitive to the speed of the oncoming traffic as they selected a gap to make a permitted left turn (Staplin, Lyles 1991; Davis, Swenson 2004). In this study, the SL of the major road (high vs. low) is used to reflect the travel speed of the vehicles. It is shown that this factor is still insignificant in explaining the differences in drivers' gap acceptance. However, the result is based on analysis of SLs ranging from 30 to $45 \mathrm{mph}$. Future study may be conducted with a wider range of speed differences,

With regard to individual characteristics, the driver's gender is significant in their gap acceptance selection. Female drivers are generally more conservative and less likely to accept a given gap than male drivers, as found in previous studies (Alexander et al. 2002; Yan et al. 2007). However, the age category is not significant. One explanation is that although elderly drivers were expected to be more conservative than younger drivers, this effect may only play a part in certain conditions, for example, with limited visibility at night time. But in our study, all data were collected in the daytime with clear visibility and elderly drivers did not have difficulty judging the gap size and complete the gap acceptance behavior. On the other hand, as we know, elderly drivers generally response and react more slowly than younger drivers, and thus if elderly drivers exhibit no significant differences from other drivers in gap acceptance, it may indicate that the left-turn maneuver posed higher risk to them. The safety of elderly drivers' gap acceptance behavior needs further investigation.

Traffic conditions at the intersection should be a major factor influencing the driver's gap acceptance behavior. In this paper, the number of rejected gaps, and the mean and total time interval of the rejected gaps by each driver, which has been rarely studied by others, are analyzed, because it is expected that these indices can be used to measure the intensity of oncoming traffic and thus may explain the effects of traffic conditions on the variation of drivers' gap acceptance behavior. The models show that as the values of these factors increase, drivers are more likely accept shorter gaps. The total time interval of the rejected gaps is the product of the number of the rejected gaps and the mean time interval of the rejected gaps. However, the model including the latter two factors yields better statistics of goodness of fit than the one including only the prior factor. This may be explained by the fact that drivers are generally not able to give accurate estimates of the total time elapsed, but are more sensitive to the number of the gaps they reject and the interval of each rejected gap. Since these factors have been rarely studied before, the findings provide some new insights into the effects of traffic conditions on drivers' gap acceptance behavior.

\section{Conclusions}

Gap acceptance theory is the basis for capacity analysis of unsignalized intersections. In practice, however, driver's gap acceptance process has usually been simplified. For instance, in some traffic simulation software, a critical gap is defined and gaps larger than it would be all accepted and gaps smaller than it would be rejected. Obviously, this does not comply with the real conditions. If a model can integrate the factors that have significant effects on the driver's gap acceptance behavior and can be put into application, it may better represent real traffic operations and yield more accurate capacity analysis. This paper focused on the gap acceptance behavior of left turning drivers from major road and a number of factors were studied which were expected to have effects on the driver's gap acceptance behavior. The major findings are listed as follows.

- Although intuitively it is thought that driver's gap acceptance behavior should be affected by the intersection layout, the modelling results show that the presence of a LTL and the number of lanes on the major road have no significant effects on the driver's probability of accepting a given gap. Meanwhile, the effect of the SL on the major road is insignificant too. Thus, the gap acceptance model can be simplified excluding these factors.

- Driver's gender also has a significant effect on the gap acceptance behavior. Female drivers are more conservative than male drivers in accepting a given gap, which is indicated by the odds ratio value of 0.55 . On the other hand, no age difference has been found in the driver's gap acceptance behavior.

- The number of rejected gaps, the mean and the total time interval of the rejected gaps are all significant in explaining the variation in the driver's gap acceptance behavior. The model with both the number of rejected gaps and the mean interval of the rejected gaps has the best model fit and is selected as the gap acceptance model. With the increase in the values of these two factors, the probability of accepting a given gap also rises up.

Further studies may be conducted on how to improve the model and apply it in practice to assist in analyzing the traffic operations and capacity at the unsignalized intersections. Data from a larger number of intersections controlling for specific features would be necessary to obtain results that are more readily transferable to other contexts. Moreover, the findings may be applied to intersection decision supporting systems to assist drivers in their gap acceptance decisions, but the effects of the system itself on drivers' behavior require thorough research in the future. 


\section{Acknowledgments}

The research described in this paper was supported by a grant from the New England University Transportation Center, a program of the United States Department of Transportation, and National Natural Science Foundation of China (Grant No. 51308092).

\section{References}

Alexander, J.; Barham, P.; Black, I. 2002. Factors influencing the probability of an incident at a junction: results from an interactive driving simulator, Accident Analysis \& Prevention 34(6): 779-792. http://dx.doi.org/10.1016/S0001-4575(01)00078-1

Beanland, V.; Lenné, M. G.; Candappa, N.; Corben, B. 2013. Gap acceptance at stop-controlled T-intersections in a simulated rural environment, Transportation Research Part F: Traffic Psychology and Behaviour 20: 80-89. http://dx.doi.org/10.1016/j.trf.2013.05.006

Becic, E.; Manser, M. P.; Creaser, J. I.; Donath, M. 2012. Intersection crossing assist system: transition from a road-side to an in-vehicle system, Transportation Research Part F: Traffic Psychology and Behaviour 15(5): 544-555. http://dx.doi.org/10.1016/j.trf.2012.05.010

Brilon, W.; Koenig, R.; Troutbeck, R. J. 1999. Useful estimation procedures for critical gaps, Transportation Research Part A: Policy and Practice 33(3-4): 161-186. http://dx.doi.org/10.1016/S0965-8564(98)00048-2

Cassidy, M. J.; Madanat, S. M.; Wang, M.-H.; Yang, F. 1995. Unsignalized intersection capacity and level of service: revisiting critical gap (with discussion and closure), Transportation Research Record 1484: 16-23.

Creaser, J. I.; Rakauskas, M. E.; Ward, N. J.; Laberge, J. C.; Donath, M. 2007. Concept evaluation of intersection decision support (IDS) system interfaces to support drivers' gap acceptance decisions at rural stop-controlled intersections, Transportation Research Part F: Traffic Psychology and Behaviour 10(3): 208-228.

http://dx.doi.org/10.1016/j.trf.2006.10.004

Davis, G. A.; Swenson, T. 2004. Field study of gap acceptance by left-turning drivers, Transportation Research Record 1899: 71-75. http://dx.doi.org/10.3141/1899-09

Devarasetty, P. C.; Zhang, Y.; Fitzpatrick, K. 2012. Differentiating between left-turn gap and lag acceptance at unsignalized intersections as a function of the site characteristics, Journal of Transportation Engineering 138(5): 580-588. http://dx.doi.org/10.1061/(ASCE)TE.1943-5436.0000368

Dissanayake, S.; Lu, J. J.; Yi, P. 2002. Driver age differences in day and night gap acceptance capabilities, IATSS Research 26(1): 71-79.

Highway Capacity Manual. 2000. Transportation Research Board (TRB), National Research Council. Washington, D.C., U.S. 1134 p.

Kaysi, I. A.; Abbany, A. S. 2007. Modeling aggressive driver behavior at unsignalized intersections, Accident Analysis \& Prevention 39(4): 671-678. http://dx.doi.org/10.1016/j.aap.2006.10.013

Laberge, J. C.; Creaser, J. I.; Rakauskas, M. E.; Ward, N. J. 2006. Design of an intersection decision support (IDS) interface to reduce crashes at rural stop-controlled intersections, Transportation Research Part C: Emerging Technologies 14(1): 39-56. http://dx.doi.org/10.1016/j.trc.2006.03.001
McGowen, P.; Stanley, L. 2012. Alternative methodology for determining gap acceptance for two-way stop-controlled intersections, Journal of Transportation Engineering 138(5): 495-501. http://dx.doi.org/10.1061/(ASCE)TE.19435436.0000358

Pollatschek, M. A.; Polus, A.; Livneh, M. 2002. A decision model for gap acceptance and capacity at intersections, Transportation Research Part B: Methodological 36(7): 649663. http://dx.doi.org/10.1016/S0191-2615(01)00024-8

Polus, A.; Lazar, S. S.; Livneh, M. 2003. Critical gap as a function of waiting time in determining roundabout capacity, Journal of Transportation Engineering 129(5): 504-509.

http://dx.doi.org/10.1061/(ASCE)0733-947X(2003)129:5(504)

Polus, A.; Shiftan, Y.; Shmueli-Lazar, S. 2005. Evaluation of the waiting-time effect on critical gaps at roundabouts by a logit model, European Journal of Transport and Infrastructure Research 5(1): 1-12.

Rakha, H.; Sadek, S.; Zohdy, I. 2011. Modeling differences in driver left-turn gap acceptance behavior using bayesian and bootstrap approaches, Procedia - Social and Behavioral Sciences 16: 739-750. http://dx.doi.org/10.1016/j.sbspro.2011.04.493

Staplin, L.; Lyles, R. W. 1991. Age differences in motion perception and specific traffic maneuver problems, Transportation Research Record 1325: 23-33.

Vasconcelos, A. L. P.; Seco, Á. J. M.; Silva, A. M. C. B. 2013. Comparison of procedures to estimate critical headways at roundabouts, Promet - Traffic\&Transportation 25(1): 43-53. http://dx.doi.org/10.7307/ptt.v25i1.1246

Yan, X.; Radwan, E.; Guo, D. 2007. Effects of major-road vehicle speed and driver age and gender on left-turn gap acceptance, Accident Analysis \& Prevention 39(4): 843-852. http://dx.doi.org/10.1016/j.aap.2006.12.006

Zohdy, I.; Rakha, H. A. 2012. Framework for intersection decision support in adverse weather conditions, Transportation Research Record 2324: 20-28. http://dx.doi.org/10.3141/2324-03

Zohdy, I.; Rakha, H. A.; Alfelor, R.; Yang, C. Y. D.; Krechmer, D. 2011. Impact of inclement weather on left-turn gap acceptance behavior of drivers, Transportation Research Record 2257: 51-61. http://dx.doi.org/10.3141/2257-06

Zohdy, I.; Sadek, S.; Rakha, H. A. 2010. Empirical analysis of effects of wait time and rain intensity on driver left-turn gap acceptance behavior, Transportation Research Record 2173: 1-10. http://dx.doi.org/10.3141/2173-01 